LHCb 2002-004

Physics

\title{
Status of the LHCb experiment
}

On behalf of the LHCb collaboration

T. Nakada

CERN, CH-1211 Geneva 23, Switzerland

and

IPHE, Université de Lausanne, CH-1015 Lausanne, Switzerland

\author{
Prisented at \\ III International Symposium on LHC Physics and Detectors \\ Chia, Sardinia, Italy \\ October 25-27, 2001
}

February 7, 2002

\begin{abstract}
$\mathrm{LHCb}$ is a dedicated experiment to study $\mathrm{CP}$ violation and other rare processes in the $\mathrm{B}$ meson system at LHC. It is designed to exploit the large sample of $\mathrm{B}_{\mathrm{d}}$ and $\mathrm{B}_{\mathrm{s}}$ mesons available at LHC by having a trigger system efficient for both leptonic and hadronic final states, particle identification capability over large momentum range and excellent decay time resolution. After the approval of Technical Design Reports of various sub-detector systems, construction of detector components has started. The experiment is planned to be ready for data taking from the beginning of the LHC operation.
\end{abstract}

\section{Introduction}

The Large Hadron Collider (LHC) [1] at CERN was designed to collide protons at a center of mass energy of $14 \mathrm{TeV}$ using the existing LEP tunnel. The 
machine is aimed to discover the Higgs particle [2], the last missing ingredient of the Standard Model, and to search for particles beyond the Standard Model, such as the Super Symmetric (SUSY) particles [3].

The production cross section of the $b \bar{b}$ quark pairs at the LHC energy is estimated to be $\sim 500 \mu \mathrm{b}$, far larger than at any existing machines. The fraction of events with b quarks, $\sigma_{\mathrm{b} \bar{b}} / \sigma_{\text {inelastic }}$, is about $6 \times 10^{-3}$ which is similar to the fraction of charm events in the present fixed-target charm experiments. Thus, LHC appears to be a very promising place to perform high precision $\mathrm{CP}$ violation measurements in B-meson decays. At $\mathrm{LHC}, \mathrm{B}_{\mathrm{s}}, \overline{\mathrm{B}}_{\mathrm{s}}, \mathrm{B}_{\mathrm{c}}^{ \pm}$and b-baryons are abundantly produced, in addition to $\mathrm{B}^{ \pm}, \mathrm{B}^{0}$ and $\overline{\mathrm{B}}^{0}$.

The goal of $\mathrm{B}$ physics in the LHC era $[4,5]$ is to determine the CKM parameters in a model-independent way and to isolate the effect of New Physics so that its characteristics could be identified. This calls for a high statistics experiment capable of studying $\mathrm{CP}$ violation with both $\mathrm{B}^{0}$ and $\mathrm{B}_{\mathrm{s}}^{0}$ systems decaying into various final states, including those with only hadrons. In order to exploit the potential of LHC, experiments need to have the following capabilities:

1. Trigger sensitive to both leptonic and hadronic final states.

2. Particle identification system capable of identifying $\mathrm{p}, \mathrm{K}, \pi, \mu$ and $\mathrm{e}$ within the required momentum range.

3. Vertex detector able to reconstruct primary and b-hadron vertices very precisely.

4. Tracking system with good momentum resolution.

In addition, a capability of reconstructing $\pi^{0}$ would enhance the potential of the experiment further. The LHCb spectrometer is designed to fulfil these requirements. In this paper, we give an overview of the detector and the status of the experiment. Some of the detector components and trigger are described in detail in these proceedings $[6,7,8,9,10]$ together with the expected physics performance of the experiment [11]. 


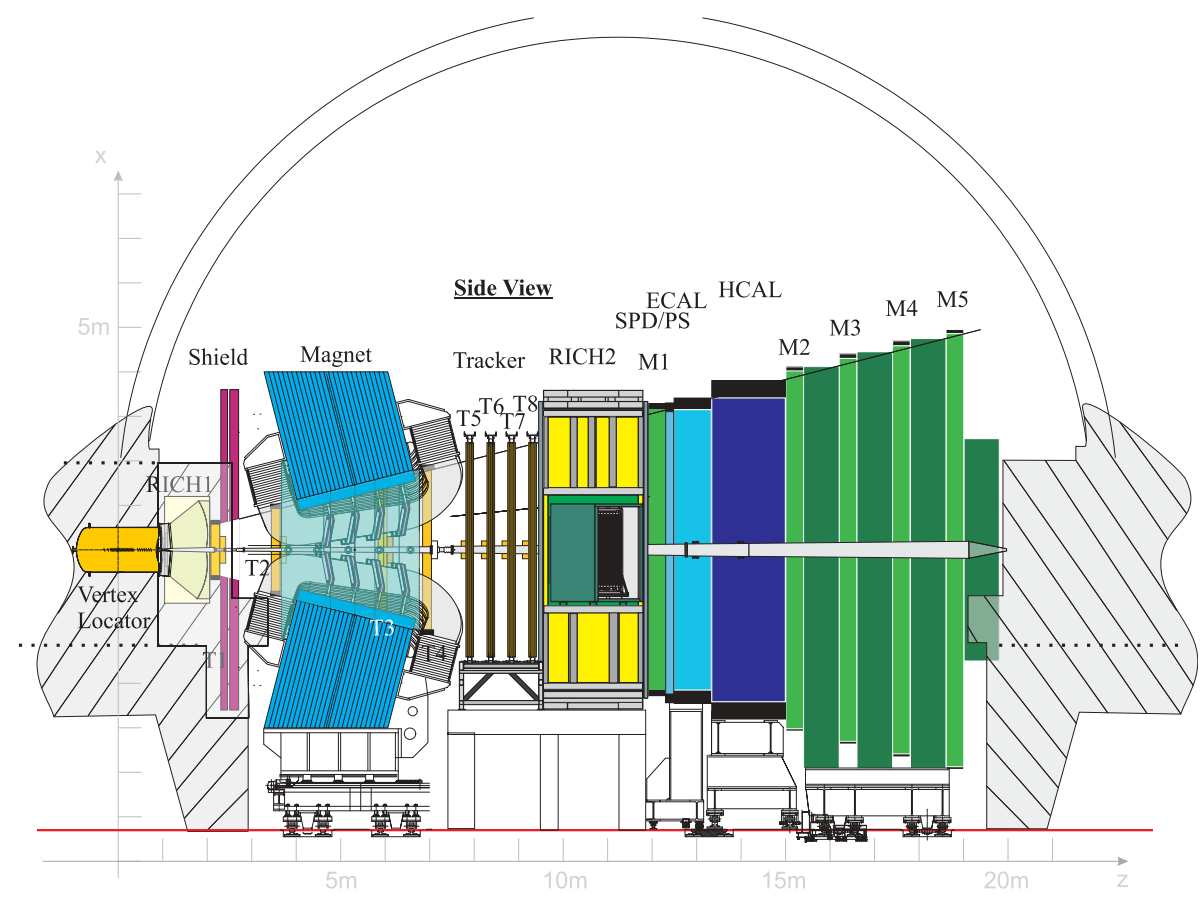

Figure 1: The LHCb spectrometer placed in the LHC Intersection-8 experimental area.

\section{LHCb Experiment}

\subsection{Overall Picture of the Experiment}

The layout of the LHCb detector [12] shown in Figure 1 resembles a typical fixed target spectrometer due to its forward geometry. It consists of a vertex detector at the intersection point (placed in "Roman Pots"), a tracking system, Ring Imaging Cherenkov Counters with aerogel and gas radiators, a large-gap dipole magnet, a calorimeter system, and a muon system. An existing LEP experimental area is reused to install the detector. The interaction point is shifted by $11 \mathrm{~m}$ from the nominal point, the centre of the experimental hall, in order to accommodate the detector elements without extra excavation.

The choice of the detector geometry is based on the fact that both the b- and $\bar{b}$-hadrons are predominantly produced in the same forward (or backward) cone at high energies. This is demonstrated in Figure 2 where the polar 


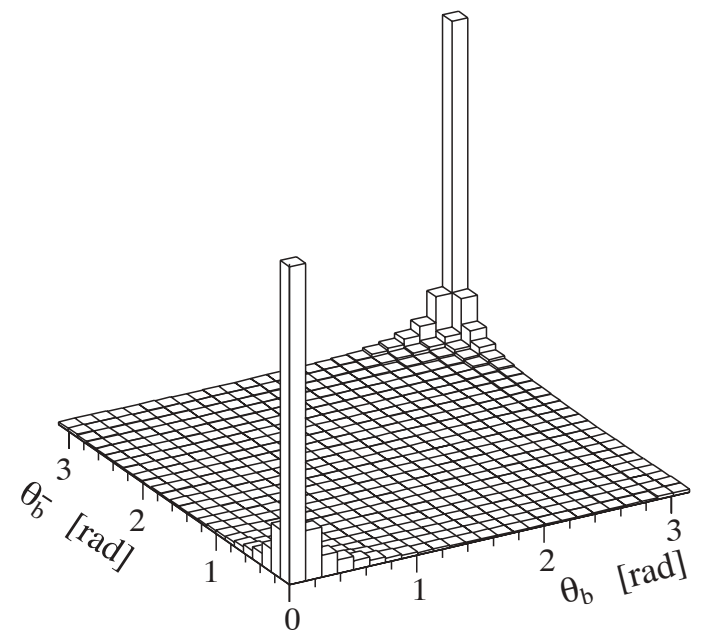

Figure 2: Polar angles of the b- and $\bar{b}$-hadrons obtained with the PYTHIA simulation programme.

angles of the $\mathrm{b}$ - and $\bar{b}$-hadrons in one event obtained with the PYTHIA event generator are shown. The polar angle is defined with respect to the beam axis in the pp center-of-mass system. Detecting both $b-$ and $\bar{b}$-hadron at the same time is essential for the flavour tag.

Further advantages of the forward geometry are:

- The b-hadrons produced in the forward direction are faster than those in the central region. Their average momentum is about $80 \mathrm{GeV} / c$, corresponding to a mean decay length of $\sim 7 \mathrm{~mm}$. Therefore, a good decay time resolution can be obtained for reconstructed b-hadrons.

- The spectrometer can be built in an open geometry with an interaction region which is not surrounded by all the detector elements. This allows a vertex detector system to be built with sensors which can be extracted away from the beam during the injection using Roman Pot technique. During the data taking, the sensors are positioned close to the beam in order to achieve a good vertex resolution.

- In the forward region, momenta are mainly carried by the longitudinal components. Therefore, the threshold values for the $p_{\mathrm{t}}$ triggers can be set low for electrons, muons and hadrons without being constrained by 
the detector requirements. This makes the $p_{\mathrm{t}}$ triggers more efficient than in the central region.

- The momentum range required for particle identification is well matched to the Ring Imaging Cherenkov (RICH) technique. The required size for the RICH counters remains affordable.

- The open geometry allows easy installation, maintenance and possible upgrade.

It is also important to note that the detector covers a region of phase space which is not looked at by the two general purpose detectors, ATLAS and CMS.

Due to its efficient trigger, the $\mathrm{LHCb}$ experiment will be able to exploit fully the physics potential of LHC at a much lower luminosity $(2 \times$ $\left.10^{32} \mathrm{~cm}^{-2} \mathrm{~s}^{-1}\right)$ than the nominal LHC luminosity $\left(10^{34} \mathrm{~cm}^{-2} \mathrm{~s}^{-1}\right)$. Therefore, the experiment will be able to start its full physics programme from the beginning of the LHC operation.

\subsection{Beam Pipe}

A large vacuum tank with a length of $1.7 \mathrm{~m}$ and a diameter of $1 \mathrm{~m}$ is placed around the interaction point to accommodate the vertex detector system with its retraction mechanics. It has a $2 \mathrm{~mm}$ thick $\mathrm{Al}$ forward exit window over the full detector acceptance. This part is followed by two conical sections: the first one is $1.4 \mathrm{~m}$ long with a $25 \mathrm{mrad}$ opening angle, and the second part is $16 \mathrm{~m}$ long with a $10 \mathrm{mrad}$ opening angle.

Except for bellows and flanges and the last $6.3 \mathrm{~m}$ of the $10 \mathrm{mrad}$ cone, the beam pipe is made of Al-Be alloy in order to reduce the radiation length. This is essential for minimising the occupancies of the tracking and RICH systems, as well as for the detection of photons and electrons. Due to mechanical and safety reasons, flanges and bellows are made of $\mathrm{Al}$.

The beam pipe section in the region of the calorimeters and muon system is no longer important for the detection of electrons and photons. It is surrounded by shielding material, thus generating background particles is no longer an issue. Therefore, stainless steel is used for this section. 


\subsection{Magnet}

A dipole magnet [13] with $\mathrm{Al}$ conductor provides a field integral of $4 \mathrm{Tm}$. The polarity of the field can be changed to reduce systematic errors in the $\mathrm{CP}$-violation measurements that could result from a left-right asymmetry of the detector.

The two pole faces form a wedge shape following the spectrometer acceptance, in order to reduce the power consumption to $42 \mathrm{MW}$. The yoke of the magnet weights $1.5 \mathrm{kt}$ and consists of many iron plates assembled together. The magnet assembly will take place in the experimental area.

\subsection{Vertex Locator (VELO)}

A total of 27 stations of silicon microstrip detectors are placed perpendicular to the beam of which 25 stations are used as a vertex detector system (Vertex Locator) $[14,8]$. The remaining two stations are dedicated for detecting bunch crossings with more than one pp interaction as part of the Level-0 trigger (pile-up veto counter).

Stations are split into two halves, covering the left and right $180^{\circ}$ sections. Each vertex detector station consists of two Si sensor planes with different strip layouts, one for $r$ and the other for $\phi$ measurements. There are total of $200 \mathrm{k}$ strips with varying sizes so that the occupancy remains below $1 \%$ everywhere. The pile-up veto counter has only $r$-measuring sensors.

Signals are read-out and stored in analog pipeline buffers for every bunch crossing, i.e. every 25 ns.

The closest distance between the active silicon area and the beam is $8 \mathrm{~mm}$. In order to cope with high radiation dose expected at this position so close to the beam, n-on-n silicon sensors are taken as baseline.

The silicon detectors are placed in Roman Pots with $250 \mu \mathrm{m}$ thick aluminium foil, which acts as a shield against RF pickup of the circulating beam bunches. In order to avoid explosion, a secondary vacuum is maintained inside the Roman Pots. During the injection and acceleration, the Roman Pot system will be moved away from the beam to avoid interference with the machine operation and accidental irradiation of the detectors. 


\subsection{Tracking}

Because of the high particle density close to the beam pipe, the LHCb tracking detector [9] is split into inner and outer systems. The boundary between the two was chosen so that the occupancy of the outer tracker does not exceed $15 \%$ at the highest point.

The outer tracking system [15] uses drift chambers based on a straw cells structure. Straws are made by winding the carbon-loaded Kapton foil. The diameter of the straw is $5 \mathrm{~mm}$ and drift-time is sampled over $50 \mathrm{~ns}$, i.e. two bunch crossings.

The inner tracking system is made from single sided p-on-n Si strip detectors with a strip pitch of $\sim 200 \mu \mathrm{m}$. Since the sensitive regions of the Si sensor are further away from the beam (several centimeters) compared with the sensors of the Vertex Locator, the problem of radiation damage is less severe.

\section{$2.6 \quad \mathrm{RICH}$}

The RICH system of the LHCb detector $[16,10]$ consists of two detectors with three different radiators in order to cover the required momentum range, 1$100 \mathrm{GeV} / c$. The first detector uses aerogel and $\mathrm{C}_{4} \mathrm{~F}_{10}$ gas as radiators. The second detector, design to identify high momentum particles, is placed after the magnet and uses $\mathrm{CF}_{4}$ as radiator.

The Cherenkov light is detected with planes of Hybrid Photon Detectors (HPD's) placed outside the spectrometer acceptance.

\subsection{Calorimeters}

The calorimeter system [17] consists of a preshower detector followed by electromagnetic and hadronic calorimeters. The primary function of the calorimeter system is to provide single-particle energy information to the Level-0 trigger. It is also used in offline to reconstruct $\pi^{0}$ from $\mathrm{B}$ meson decays such as $\mathrm{B}_{\mathrm{d}} \rightarrow \rho^{0} \pi^{0}$. It serves as the initial part of the muon filter system.

The cells of the Preshower detector are made as two scintillator plates sandwiching a $14 \mathrm{~mm}$-thick lead plate. The cell size of the preshower detector is matched to the module size of the electromagnetic calorimeter. 
For the electromagnetic part, a Shashlik calorimeter is used since a modest energy resolution of $\sim 10 \%$ is needed. It is $6.3 \mathrm{~m}$ tall, $7.8 \mathrm{~m}$ wide and $83.5 \mathrm{~cm}$ long corresponding to $25 X_{0}$. The cell size of a module changes from $4 \times 4 \mathrm{~cm}^{2}$ in the zone closest to the beam pipe, to $6 \times 6 \mathrm{~cm}^{2}$ in the intermediate zone, and to $12 \times 12 \mathrm{~cm}^{2}$ in the outer most zone, minimizing the number of modules without deteriorating the performance. A similar electromagnetic calorimeter system is successfully operating in HERA-B.

The hadron calorimeter is based on a scintillating tile design similar to that used in the ATLAS experiment with a reduced energy resolution of $\sim 80 \%$. It is $6.8 \mathrm{~m}$ tall, $8.4 \mathrm{~m}$ wide and $165.5 \mathrm{~cm}$ long corresponding to $5.6 \lambda_{\mathrm{I}}$. There are only two cell sizes for the hadron calorimeter modules; $13 \times 13 \mathrm{~cm}^{2}$ and $26 \times 26 \mathrm{~cm}^{2}$.

Since it is used in the Level-0 trigger, energy information is digitized every $25 \mathrm{~ns}$ and delivered to the trigger. Part of the trigger electronics is integrated in the readout electronics [6]. Identical readout electronics are used for the electromagnetic and hadronic calorimeters.

\subsection{Muon}

The muon system [18] consists of four layers of hadron absorbers and five muon stations. The first station is placed in front of the first absorber layer, which is the calorimeter system. The three other absorbers are made of iron plates, each of them $80 \mathrm{~cm}$ thick. Three muon stations are placed between the absorbers and the fifth muon station is placed behind the last absorber, shielded with $20 \lambda_{\text {I }}$ of material. A smaller shielding plate behind the last station protects the chambers from the particles produced by the proton beam in the accelerator.

For the muon stations, Resistive Plate Chambers are used in the region where the charged particle rate is below $1 \mathrm{kHz} / \mathrm{cm}^{2}$. In the region with a charged particle rate from $1 \mathrm{kHz} / \mathrm{cm}^{2}$ to $100 \mathrm{kHz} / \mathrm{cm}^{2}$, Multi Wire Proportional Chambers (MWPC's) are used. In the small region of the first muon station close to the beam pipe, where the charged particle rate exceeds $100 \mathrm{kHz} / \mathrm{cm}^{2}$, triple-GEM chambers or MWPC's with asymmetric gas gap are being considered.

Muon $p_{\mathrm{t}}$ information from the muon system is used in the Level-0 trigger and part of the trigger electronics is integrated in the readout electronics [7]. 


\subsection{Trigger}

The LHCb trigger is divided into four decision levels. The Level-0 decision is based on high- $p_{\mathrm{t}}$ hadrons, electrons or $\gamma$ found in the calorimeter system [6] or muons found in the muon system [7]. Information on those candidates are sent to the Level-0 Decision Unit. The number of primary vertex candidates is determined using the pile-up veto counters in the Vertex Locator and sent also to the Level-0 Decision Unit. Based on all the information, the Level-0 Decision Unit makes an overall Level-0 decision. Events with multiple pp interactions are discarded. Rejecting background in the reconstructed final states and obtaining a correct flavour tag are much more difficult in those events. The Level-0 trigger provides a modest reduction of minimum bias events by a factor of $\sim 10$.

At Level-1 [8], data from the vertex detector are used to select events with multiple vertices. In addition, those $p_{\mathrm{t}}$ measurements made by Level-0 can be combined with the tracks having large impact parameter found in the Vertex Locator. Level-1 gives a further reduction factor of $\sim 25$ for minimum bias events.

After a positive decision of the Level-1 trigger, data are read out to an event buffer. Hereafter, all the detector information is in principle available for the trigger decision. At Level-2, a further enhancement of events with b-hadrons is achieved by combining different detector components, e.g. by adding momentum information from the main tracking system to the impact parameter calculation with the Vertex Locator. At Level-3, the trigger decision is made by reconstructing the decays.

\subsection{Computing}

The LHCb computing project consists of the online system (Data Acquisition and Experiment Control Systems), offline software and computing infrastructure.

The Data Acquisition System is built from readout control, readout network and event filter farm. The Level-1 trigger accepts events with a rate of $40 \mathrm{kHz}$. The detector information is then sent to the event filter farm in one go. Therefore, higher level triggers can access all the detector information. A complete architecture for the Data Acquisition System has been designed and simulated. It is very simple and scalable, essential for maintenance and upgrade. 
A unique framework of the Experimental Control System will be used to control and monitor the whole experiment including the data acquisition. This will ease the maintenance and operation.

Except for the simulation software where GEANT3 is still used, a framework based on the Object Oriented Technology written in $\mathrm{C}++$, GAUDI, is implemented for the LHCb offline software. Within this framework, many routines written in FORTRAN are still used for reconstruction and analysis. They are gradually replaced by routines developed using new software technology.

In collaboration with other LHC experiments, LHCb is developing regional computing centres which will form the necessary computing infrastructure for the reconstruction and analysis of large amount of data. Already now, significant amount of simulated data are produced outside of CERN.

\section{Status of the Experiment}

After the submission of the Technical Proposal [12] in January 1998, the project was approved in October 1998. By the time of this conference, the Technical Design Reports for the Magnet, Calorimeters, and RICH have been approved. The Technical Design Reports for the Vertex Locator and Muon System are recommended for an approval and the Technical Design Report for the Outer Tracker System has just been submitted.

By the end of 2001, the Online Technical Design Report will be submitted, followed by those for the Trigger and Inner Tracker by the end of 2002. The Technical Design Report for the Computing will be submitted after 2002.

Construction works have started in the areas of magnet and calorimeter modules. While the magnet components are being constructed by the industry, calorimeter modules are being built by the collaborating institutes. Production of the readout electronics for the electromagnetic and hadron calorimeters will start soon as well.

Due to the relatively small size of the detector, there is still some time left before starting the construction of the rest of the components, in particular the Vertex Locator, RICH-1 and tracking system. Therefore, our priority has been put to reoptimize these three detector components in order to reduce the material budget. The detector material up the the last tracking station amounts to $60 \%$ of $X_{0}$ and $20 \%$ of $\lambda_{\text {I }}$. In addition, the beam pipe contributes to the material budget. Reduction of material in this region is very important 
for the efficient reconstruction of $\gamma, \mathrm{e}^{ \pm}$and charged hadrons with a good momentum resolution.

Already a significant improvement was made in the beam pipe design described in this paper compared with the previous design where the pipe was made from $\mathrm{Al}$ with stainless steel flanges and bellows. Some more optimization can be done by adjusting the position of the flanges and bellows.

RICH-1 material budget can be improved by producing the spherical mirror with Be or a composite material. For the Vertex Locator, various possibilities such as reducing the number of stations, improving the design of $R \& F$ shield and reducing the thickness of the Si sensors, are being investigated.

For the tracking system, the most promising way is to reduce the number of stations drastically by removing all the stations in the magnet. This requires a new tracking strategy different from what has been developed so far based on the HERA-B algorithm. The new strategy is close to what was used in the fixed target experiments. Preliminary studies show encouraging results that a similar tracking efficiency can be achieved without the tracking stations in the magnet.

With all those ingredients, we aim to make a substantial reduction of the material budget. The complete design of the reoptimized detector should be finalised before the end of 2002 in order to be ready for data taking as soon as the LHC becomes operational.

\section{Summary}

The LHCb experiment will fully exploit the large sample of $\mathrm{B}_{\mathrm{d}}$ and $\mathrm{B}_{\mathrm{s}}$ mesons produced at LHC by measuring $\mathrm{CP}$ asymmetries in many different decay modes with leptons and hadrons in the final states. This will cleanly isolate the $\mathrm{CP}$ violation effect due to the Standard Model and allow the effect produced by physics beyond the Standard Model to emerge. Essential features of the experiment are particle identification, efficient trigger and excellent decay time resolution. $\mathrm{LHCb}$ aims to be ready to take data when the LHC machine becomes operational. The project proceeds according to the schedule and construction of some detector components have started. Due to its efficient trigger, the experiment will perform its full physics programme with a luminosity of $2 \times 10^{32} \mathrm{~cm}^{-2} \mathrm{~s}^{-1}$. This is very well matched to the startup plan of the machine. 


\section{References}

[1] L. Evans: Machine Status, In these proceedings

[2] K. Lassila Perini: Higgs physics at LHC (SM, MSSM), In these proceedings

[3] S. Asai: $S U S Y$ al $L H C$, In these proceedings

[4] A. Ahmadov et al.: Proceedings of the Workshop on Standard Model Physics (and more) at the LHC, CERN 2000-04

[5] T. Nakada: B physics at $L H C(b)$, Proceedings of A NATO Advanced Study Institute 55th Scottish Universities Summer School in Physics, 2001

[6] U. Marconi: Level-0 Calorimeter Trigger, In these proceedings

[7] O. Leroy: Level-0 Muon Trigger, In these proceedings

[8] F. Teubert: Level-1 Trigger, In these proceedings

[9] M. Needham: Tracking, In these proceedings

[10] P. Soler: Particle Identification, In these proceedings

[11] P. Koppenburg: Physics performance in LHCb, In these proceedings

[12] S. Amato et al., LHCb Collaboration: LHCb Technical Proposal, CERN/LHCC/98-4

[13] S. Amato et al., LHCb Collaboration: LHCb Magnet Technical Design Report, CERN/LHCC/2000-7

[14] S. Amato et al., LHCb Collaboration: LHCb VELO Technical Design Report, CERN/LHCC/2001-11

[15] S. Amato et al., LHCb Collaboration: LHCb Outer Tracker Technical Design Report, CERN/LHCC/2001-24

[16] S. Amato et al., LHCb Collaboration: LHCb RICH Technical Design Report, CERN/LHCC/2000-37 
[17] S. Amato et al., LHCb Collaboration: LHCb Calorimeters Technical Design Report, CERN/LHCC/2000-36

[18] S. Amato et al., LHCb Collaboration: LHCb Muon System Technical Design Report, CERN/LHCC/2001-10 